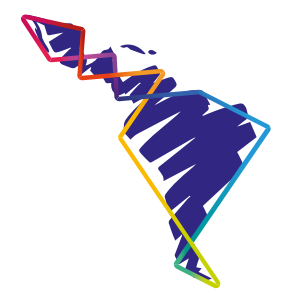

\title{
Un acercamiento sensible al estudio de las migraciones en la poesía de Alejandra Solórzano Castillo
}
A sensible approach to the study of migrations in the poetry of Alejandra Solórzano Castillo

\section{Uma abordagem sensata ao estudo das migrações na poesia de Alejandra Solórzano Castillo}

\section{Silvia Elena Guzmán Sierra}

\begin{abstract}
Resumen
A manera de experimento sensible, el presente artículo se acerca a la subjetividad de una migrante centroamericana. Usando como herramienta filosófica la racionalidad poética de María Zambrano, el acercamiento se hace a través de una selección de poemas de la autora Alejandra Solórzano Castillo, contenidos en los libros Todo esto sucederá siempre (2015) y Detener la historia (2017). Con la experiencia de los textos poéticos, se acerca también a la historia de esta Mesoamérica doliente.
\end{abstract}

Palabras clave: Migraciones, itinerancia, dolor, cuerpo, mujer, reencuentro.

\begin{abstract}
As a sensible experiment, the present article approaches the subjectivity of a Central American migrant. Using María Zambrano's poetic rationality as a philosophical tool, the approach is made through a selection of poems by the author Alejandra Solórzano Castillo, contained in the books Todo esto Sucederá Siempre (2015) (translated as All this will Always Happen) and Detener la Historia (2017) (translated as Stop History). With the experience of poetic texts, an approximation is also had with the history of this suffering Mesoamerica.
\end{abstract}

1 Máster en Derechos Humanos y Educación para la Paz de la Universidad Nacional, Costa Rica. Bachiller en Relaciones Internacionales y con estudios en Filosofía de la misma universidad. Se desempeña como consultora e investigadora en temas de Derechos Humanos, especialmente en materia de cultura, género y juventudes, Costa Rica. ORCID: https://orcid.org/0000-0002-3146-9456 
Keywords: Migrations; Itinerancy; Pain; Body; Woman; Reunion.

\section{Resumo}

Como um experimento sensivel, este artigo aborda a subjetividade de um migrante da América Central. Utilizando a racionalidade poética de María Zambrano como ferramenta filosófica, a abordagem é feita por meio de uma seleção de poemas da autora Alejandra Solórzano Castillo, contida nos livros, Todo esto Sucederá Siempre (2015) (traduzido como Tudo isso Acontecerá Sempre) e Detener la Historia (2017) (traduzido como História da Parada). Com a experiência de textos poéticos, ele também aborda a história desse sofrimento na Mesoamérica.

Palavras-chave: Migrações; Itinerante; Dor; Corpo; Mulher; Reunião.

\section{Advertencia}

El siguiente texto no es, solo, un ejercicio académico, aunque parta de estas rigideces para hacerse un espacio en el mundo de lo escrito. No es, tampoco, un análisis filológico o literario, carezco de las habilidades técnicas para tal emprendimiento. Mucho menos es un recuento de hechos o una historiografía. Lo siguiente es un híbrido, un ejercicio reflexivo y dialéctico entre la literatura, la filosofía y la ciencia social, y como híbrido carece de un objetivo particular para alguna de estas áreas del conocimiento. ${ }^{2}$ Por tanto, a las siguientes páginas las ocupan tres intensiones.

Número uno, mi afán "sororario" por reconocer en vida la historia de una mujer, a la que tengo el privilegio de llamar amiga. Entiendo que la lucha por la igualdad dentro de un sistema patriarcal parte del reconocimiento y la visibilización de las vidas y quehaceres de todas aquellas personas que no sean "hombres" y que tal visibilización y reconocimiento debe hacerse desde todos los espacios posibles, desde cada acción concreta. Por todo ello, el presente es un reconocimiento al tránsito de la poeta Alejandra Solórzano Castillo y, con ello, a la vida de tantas mujeres centroamericanas que han caminado por esta Mesoamérica doliente.

La segunda intención es el tratamiento sensible de eso que se ha denominado el "fenómeno de las migraciones", específicamente las migraciones intrarregionales en Centroamérica durante los conflictos armados de los últimos treinta años del siglo XX. Según el Primer Informe Estado de la Región en Desarrollo Humano Sostenible (1999), el flujo de personas migrantes a lo interno de la región para las décadas del 70, 80 y 90 fue de 95908 (74.1\%), 80140 (65.5\%) y 93281 (64.1\%), respectivamente; datos que evidencian el énfasis en los movimientos migratorios intrarregionales (Proyecto Estado de la Región, 1999).

2 Este texto fue un ir y venir, hecho dialógico, ente Alejandra y yo.

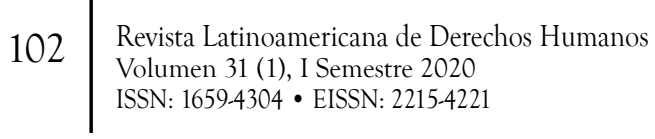


Las razones políticas y económicas de tales movilizaciones son heterogéneas y complejas, las personas solicitantes de refugio político se desplazaban hacia el país que parecía más afín a sus ideales políticos, lo que les obligaba a transitar entre unos y otros Estados centroamericanos durante los años de conflicto. Hubo, también, quienes se desplazaron por el compromiso en la organización de la resistencia y la militancia regional, este es el caso de la familia de Alejandra, quienes, en palabras de la poeta, emprendieron una itinerancia: salieron de Guatemala en 1974 y regresaron al país en 1994, veinte años de camino y movimiento entre Guatemala, Costa Rica, Nicaragua y México.

Sobre este tipo de migración, existen muy pocos estudios y aproximaciones; es mi intención, por tanto, provocar el estudio de esta temática. Provocación que no es el central contenido del presente trabajo, por lo que no me detendré en las dimensiones estadísticas, históricas o sociopolíticas del "fenómeno". Mi compromiso con quien lee es acercarle a este contexto desde lo sensible. Será decisión personal de quienes leen, si la inquietud alcanza limites, la posibilidad de un estudio de tales dimensiones.

La tercera intención surge como una motivación metodológica. En palabras de Edgar Morín, la poesía "nos introduce en la dimensión poética de la existencia humana" (Morín, 2002, p. 47), lo cual pareciera en principio redundante y, sin embargo, es un enunciado sumamente profundo. Que la poesía tenga la capacidad de introducirnos en la dimensión poética de la existencia afirma que hay una dimensión poética en la existencia del ser humano, es decir, la poética de la vida es dimensión y no objeto concreto (la poesía). Dimensión la cual podemos pasar por alto y olvidar, o acceder decididamente para el descubrimiento de algunas manifestaciones de la existencia humana. Es decir, la poética de la existencia no es el objeto de estudio de alguna disciplina del conocimiento (literatura o filología), sino un campo de las manifestaciones del ser.

La poesía devienen, entonces, en llave, en método para la investigación de las cualidades poéticas de la existencia. Por eso me acerco metodológicamente al análisis de las migraciones desde la poesía, me acerco a modo de experimentación, con la finalidad de descubrir, en los textos de Alejandra, la sensibilidad desvalorada en los estudios clásicos sobre migraciones.

\section{Una investigación sensible}

Por sensible entenderemos todo aquello que es percibible a los sentidos, hablo de una investigación estética, pero más allá del formalismo filosófico que supondría la incorporación de tal categoría, hablo de una posibilidad que hemos pasado por alto: la posibilidad de la identificación sensorial entre los seres humanos y de nuestro ser 
con el medio que nos rodea. Se propone, así, trascender el lugar del claustro académico, rodeado de cúmulos de datos estadísticos, discusiones categoriales, síntesis y propuestas desde el escritorio, para sacar a relucir la pluralidad de voces de quienes protagonizan el fenómeno migratorio.

Las ideas revolucionarias y reformistas, las críticas, han partido de ideales abstractos, aunque pretendidos concretos, formulados desde la racionalidad occidental moderna. Estos mismos se entienden como conceptos con características puras y específicas que intentan aprehender la realidad. Estos ideales han sido formulados en lo pulcro y limpio de las disciplinas filosóficas, políticas y económicas, desde los centros del saber (sean estos hegemónicos o emancipatorios) y, sin embargo, en los últimos siglos no hemos avanzado hacia una verdadera consciencia en pro de la dignidad de todas las personas. Carecemos de empatía y asombro, nos sobran explicaciones y comodidad. Estamos en el autoconfinamiento de la tranquilidad de la razón. Hemos vetado el mundo de lo sensible.

Necesitamos pensar de maneras disimiles e irregulares, de maneras en las que el afán egoísta por el conocimiento (saber-poder) se transforme en un afán empático por la comprensión de las realidades de las otras personas.

Como dice Francisco Soler -discípulo de Ortega y Julián Marías-, "pensar no es andar a zarpazos (Zugriff) con las cosas cercanas y lejanas; pensar no es meter en jaulas, agarrar, asir, prender, imponer, aplastar (Begriff). Ya el Ortega joven había clamado: 'salvémonos en las cosas'". Es decir, frente al pensar que usa el concepto (Begriff) hay un pensar diferente: el fenomenológico, tal como lo entiende Heidegger en su plena madurez. Cita Soler: "Así comprendida, la fenomenología es un camino que conduce allá abajo, ante (ein Weg der hinführt vor...); y que se deja mostrar eso ante lo cual es conducida (und sich zeigen lässt das wovor es gefürth wird). Esta fenomenología es una fenomenología de lo inaparente. ${ }^{3}$ (Guerra, 2008 p. 10)

Es preciso, desarrollar investigaciones que busquen en los confines del saber nuevas formas de presentar la realidad, formas variadas y sensibles que no se comuniquen desde la frialdad de la investigación cuantitativa, ni la pretendida objetividad de "cierta" investigación cualitativa. La transformación de los procesos sociales de esta actualidad globalizante exige procesos reflexivos y contextualizados, exige creatividad y creación, pero principalmente exige escapar a las ideas absolutas de la materialidad. Exige, por tanto, un pensamiento poético de la existencia.

3 No pasaré por alto la polémica respecto a la relación de Heidegger con el nacionalsocialismo alemán, en consecuencia con la fenomenología heideggeriana, las cosas deben de ser mostradas; sin embargo, esta polémica está muy lejos de mi objetivo en el presente artículo por lo cual tampoco me detendré en ella. 
El pensar ... es el decir poético originario, que precede a toda poesía, pero también es el elemento poético del arte, en la medida en que éste llega a ser obra dentro del ámbito del lenguaje. Todo lenguaje poético, tanto en este sentido amplio como en el más estricto de lo poético, es en el fondo un pensar. La esencia poética del pensar guarda el reino de la verdad del ser. (Guerra, 2008, p.10)

En este sentido, traigo a colación la razón poética de María Zambrano, la cual anuncia que es lo petico la única capacidad de comunicar lo inefable, lo indecible por carnal y humano, la razón poética es la oscuridad y creatividad del sueño, lo sentido más allá de todo sentido, la razón última por encima de toda razón (Martín, s. f.).

El conocimiento poético se logra por un esfuerzo al que sale a mitad de camino una desconocida presencia, a mitad de camino porque el afán que busca esa presencia jamás se encontró en soledad, en esa soledad angustiada que tiene quien ambiciosamente se separó de la realidad. A ése difícilmente la realidad volverá a entregársele. Pero a quien prefirió la pobreza del entendimiento, a quien renunció a toda vanidad y no se ahincó soberbiamente en llegar a poseer por la fuerza lo que es inagotable, la realidad le sale al encuentro y su verdad no será nunca verdad conquistada, verdad raptada, violada; no es alezeia, sino revelación graciosa y gratuita; razón poética. (Zambrano, 206, p.119)

Desde la ausencia de vanidad y sin buscar verdades absolutas, salgo al encuentro de diecisiete poemas de Alejandra Solórzano Castillo contenidos en los libros Detener la historia (2015) y Todo esto sucederá siempre (2017), a fin de encontrar la ensoñación, la verdad subjetiva, la oscuridad desgarradora, de una mujer que crece y se transforma con la historia centroamericana. Y digo salgo al encuentro no solo en el sentido metafórico de mi posición dialógica ante el texto, sino también porque fue Alejandra quien "los colocó en el camino" y no yo quien los escogió para los fines concretos de este experimento.

La invitación general es a pensar la realidad social, y en este caso concreto las migraciones, desde lo poético, desde una razón poética que no acapara, cuantifica o clasifica motivos de movilidad, circunstancias originarias, rutas de tránsito, relaciones políticas intrarregionales, sino que, de previo, se coloca a oscuras para el decir de una persona migrante.

La intención ya no metodológica, sino ética es escuchar sin interpretar a partir de la racionalidad moderna, lo que tengan que decir quienes viven "las realidades que estudiamos". 


\section{Una investigación para la dignidad}

Un esfuerzo por traer a la luz la dimensión poética de la existencia no tendría sentido, si el objetivo de esta fuera únicamente llenar de belleza las investigaciones sociales. Lo realmente valioso que surge de este esfuerzo es que tenga el propósito de generar caminos para la dignidad. Las investigaciones sociales y filosóficas producen conocimiento estéril, si no se evocan de lleno a la búsqueda de la dignidad.

Por tanto, cuando hablamos de dignidad (desde la perspectiva crítica de los derechos humanos), entenderemos que una vida digna es aquella en que las necesidades materiales e inmateriales son satisfechas y garantizadas. Sin embargo, estas garantías no se fijan en el tiempo, ni llegan a un punto acabado, sino que el transcurrir cambiante de la realidad social las necesidades materiales e inmateriales se modifican naturalmente. Ahora bien, es importante resaltar que estas necesidades se modifican por la tendencia al cambio de la vida en sociedad y no por el valor capitalista del consumo. Una vida digna además entiende que la dignidad debe ser para todas las personas, por lo tanto, el consumo y la competencia neoliberales quedan fuera de cualquier concepción crítica de dignidad. Hablar de dignidad es siempre hablar de derechos humanos para todas las personas.

Dice Joaquín Herrera Flores:

Los derechos humanos, más que derechos "propiamente dichos" son procesos; es decir, el resultado, siempre provisional, de las luchas que los seres humanos ponen en práctica para poder acceder a los bienes necesarios para la vida. (Herrera, s. f., p. 22)

Buscar la dimensión poética es, desde mi perspectiva, una lucha por un bien necesario para la vida. Como antes se mencionó, la dimensión poética de la vida es una especificidad del ser humano, una forma de manifestación y, por tanto, ocultarla o restringirla sería una violación a la naturaleza del ser. En concreto, se niega el "bien" inmaterial de la sensibilidad si no se da voz a esa sensibilidad en la comunicación de los fenómenos sociales.

Podrá ponerse en duda que la sensibilidad tenga la valía de un derecho como el derecho a la vida o a la vivienda; sin embargo, pensar en la posibilidad de un mundo en el que la sensibilidad (en los términos de referencia de este artículo) sea un bien fundamental, supone un mundo más libre e igualitario. Parto, además, de la concepción clásica de que los derechos humanos son universales, inalienables e interdependientes. 
De manera específica, considero que dar una mirada desde la dignidad y, por tanto, desde la sensibilidad al fenómeno de las migraciones habilita un reconocimiento empático de la condiciones de vida de las personas migrantes. En la capacidad del reconocimiento sensible de una historia de vida se generan ideas de lucha solidaria y dignidad colectiva.

\section{Una niña centroamericana, una niña revolucionaria, una niña migrante- itinerante que se busca: Alejandra}

Rosa María Castillo Villatoro y Luis Enrique Solórzano Loaiza, junto con sus dos hijas mayores, Ivonne e Ixmucané, llegan a Costa Rica en 1974 buscando refugio, provenientes de la guerra en Guatemala. Amanda y Alejandra, las dos hijas menores, nacen en Costa Rica. En el recuerdo y la nostalgia queda un país en conflicto, un país que, en palabras de Alejandra, vivía los años más sangrientos de la guerra. Ese recuerdo es cicatriz y mordedura.

Un nombre turbulento, una cicatriz.

El viento que entra y me asedia

en el centro de mi cuarto

como un trazo meditado y sin forma

sueño de eso que soy o que tal vez no:

la caída,

el eco,

trecho entre golpe de ala

y ala carcomida.

La distancia

es mi corazón de Pom,

una danza oscilante

humo en tormento y alivio.

El espejismo de mis piernas

que ese nombre quiebra e instaura al final

de los pasillos.

Un falso curativo

Heredad de todos mis objetos,

crueles monumentos

animales tristes de memoria.

La distancia es una niña profanando el pasado.

El único olor que embiste y aviva

promesas ingratas

una nube pequeña 
doliente.

Una cicatriz que prolonga mi sombra

Ese nombre incesante

que sube por mis remos,

amuleto y escondite imposible

para esta mordedura. (Solórzano, 2015, p. 45)

En 1983, el padre de Alejandra, apoyado por Rosa María decide migrar a Nicaragua. Allí en 1987, Luis Solórzano decide incorporarse al Ejército Guerrillero de los Pobres (EGP) de Guatemala. Alejandra, su madre y hermanas permanecen en Nicaragua en un colectivo de niños y niñas, hijas de militantes del EGP, fracción de la Unidad Revolucionaria Nacional Guatemalteca (URNG). Sin embargo, sus hermanas mayores deciden incorporarse a la lucha armada; Ivonne, se incorpora a la guerrilla a los 19 años y se va a las montañas guatemaltecas en 1990. Ixmucané, la segunda de las hijas, se une a la guerrilla en la lucha armada poco tiempo después de que su padre se incorporó a las fuerzas del EGP.

\section{Reconstrucciones \\ a Luis Solórzano \\ I}

Venís a explicarme, con tus siete años, cómo se lavan las tumbas de toda una ciudad. - ¿Son muchas? pregunto. -Mira el cielo, respondes para dejarme otra vez en silencio, con mis ojos atados a tus pies diminutos. Me vuelvo pez. Vos también. Observo cómo embestís la calle honrando con tu trabajo de niño los epitafios del casco viejo de la ciudad.

II

Vuelvo a hincarme frente la cama y repaso la lección de la escuela mientras el cuadernito soviético recibe mis garabatos en una noche de 29 grados de Nicaragua. Es tarde. A esta edad es difícil comprender las horas. Siento vergüenza de quedarme dormida y no esperar a que regreses. (Solórzano, 2015, p. 19)

Para el ascenso al poder en Nicaragua de la Unión Nacional Opositora, con su líder Violeta Barrios en 1990, Alejandra, Amanda y su madre, Rosa María Castillo Villatoro, deben migrar nuevamente, pues su familia ya estaba fuertemente involucrada al EGP y no era posible permanecer en Nicaragua con la toma de posesión de poder de un gobierno reaccionario.

Los años de 1990 a 1994 son convulsos, de constantes movimientos y de cambios de residencia entre el Estado de México y Chiapas, específicamente entre Comitán y San Cristóbal. Mientras Amanda, la tercera de las hermanas permanece estudiando enfermería en un convento en Distrito Federal, Rosa María solicita a su organización 
un cambio de funciones por el que se desplazan a Chiapas. Durante ese período, Rosa María trabajaba con el EGP y con el colectivo de Mujeres de Mamá Maquín, organización de mujeres campesinas guatemaltecas que buscaba un retorno seguro y acceso a tierras en su país (Guatemala).

En ese entonces era usual que la organización guerrillera para la que militaba Rosa María ofreciera un estipendio de manutención para las familias organizadas; sin embargo, el apoyo fue mermando poco a poco hasta prácticamente desaparecer, a pesar de las preguntas y reclamos de la madre. Alejandra y Rosa María pasaron períodos de hambre y vulnerabilidad, debido a que estando en una situación de compartimentación, por seguridad política, no es posible recurrir o solicitar ayuda a nadie que no sea de la organización.

Alejandra cuenta que entre los doce y trece años, tiempo en que estuvo en Comitán, su madre, procuraba entrenarla para buscar rutas diferentes para volver de la escuela a casa. Rutinas de seguridad para quedar sola en casa mientras Rosa María debía cumplir tareas por días o semanas, sin poder regresar o comunicarse con sus hijas. Alejandra debía resistirse a cualquier invitación de familias vecinas dispuestas a ofrecer amablemente comida o compañía para así evitar preguntas comprometedoras que develaran información sobre ellas (la familia) y la organización.

El silencio era un lugar seguro. Recuerda horas compartidas con su madre por la noche quemando discretamente papeles y documentación en el patio de la casa. No contestar el teléfono, saber después de qué cantidad de timbrazos levantar el auricular, saber qué contestar en caso de preguntas o llamadas "aparentemente" equivocadas. Recuerda haber recibido "compañeros de paso", gente de la organización que posiblemente pasaría una noche o días en casa de ella, sin hablarles, hacer preguntas o interactuar lo menos posible. La ayuda económica escaseaba y además del hambre la vulnerabilidad pasaba por la imposibilidad de moverse en caso de emergencia.

Alejandra me dice que dormía con un cuchillo debajo de su almohada, a veces temía -era una posibilidad-, que su madre ya no regresara. Tenía 12 años.

Después de verse en el cumplimiento de tareas cada vez más continuas y prolongadas por días, Rosa María tuvo que verse obligada a sacar a su hija de la escuela y llevársela consigo a algunas tareas. Aunque esto implicase otro tipo de riesgo, Alejandra comenta que quizá esto era lo mejor ante la imposibilidad o la impotencia de estar vulnerables y separadas. Entre estos movimientos, me dice, que una vez pasó escondida con su madre por horas dentro de un temazcal ${ }^{4}$ en desuso y, en otra ocasión,

4 Un temazcal es una edificación pequeña hecha de barro que se usa para la sanación de las personas. En ella se hierven y queman plantas medicinales generando calor que provoca la sudoración. Es a través del sudor que 
escondidas debajo de una mesa sencilla en una vivienda extremadamente sencilla en una comunidad a donde entraron soldados.

En 1993 regresan al Estado de México, pues Rosa María decide, por sobrevivencia, desvincularse de la militancia, debido lánguido apoyo económico y con el fin de poder conseguir un empleo. En esos mismos años, Luis decide separarse de Rosa María. También esa desvinculación con la militancia trajo consigo años duros de vulnerabilidad y valentía para todas, en un horizonte lejano al país de origen, sin la seguridad y los vínculos que en algún momento podrían haber dado la organización y los escasos medios para la sobrevivencia.

Malitzín Tenépatl

Seré obsequiada al señor Quetzalcóatl

Seré obsequiada a los señores de Mayab

Seré obsequiada al foráneo Hernán Cortés

Seré obsequiada al capitán Alonso Hernández Portocarrero

Seré obsequiada al hidalgo Juan Jaramillo

Seré obsequiada a Diego Rivera

Seré obsequiada a los historiadores

filólogos

políticos.

A cada boca seré un obsequio

cuando me llamen

Malinche

para decir

vendida

traidora

servil

interesada

puta

Seré un obsequio

hoy

mañana

y los días venideros

siempre a la víspera

de dejar de ser

la persona se limpia y sana. 
y antes de presentarme ante mi última dueña:

Nan Kemé

Nanita Muerte. (Solórzano, 2015, p. 47)

En el Estado de México son acogidas por una familia con la que habían trabajado en una de las casas para niños y niñas, hijas de militantes. Rosa María encuentra trabajo como maestra, con el tiempo se reencuentran con algunos de los vínculos afectivos de la organización y con la mayor de las hijas, Ivonne, quien baja de la montaña para parir a su primer hijo. Más adelante, en 1993, hacen su última movilización interna al Distrito Federal de México. En 1994, dos años antes de la firma del Acuerdo de Paz Firme y Duradera, las tres (Amanda, Alejandra y Rosa María) regresan a Guatemala. Dice Alejandra:

En el año noventa y cuatro fue que, por primera vez, fui a Guatemala. Para mí esto significó en realidad un regreso porque todo este camino, esta itinerancia, estaba relacionada con la historia de Guatemala. Fue en el año noventa y cuatro que regresamos a Guatemala, aunque yo nunca hubiese puesto un pie ahí antes. (Solórzano, comunicación personal, marzo 2018)

Alejandra tenía 14 años y hasta esa época se vuelve a reencontrar con sus hermanas mayores.

El retorno es un espejo borroso Malitzín,

una siempreviva petrificada,

un viento preso.

El regreso infertilidad de la calma,

la lluvia del silencio,

las ciénagas del terror en que todos mojamos los pies.

Somos dueños aún de esta tierra,

aves del insomnio

y el pasado nos dice

que no hemos terminado de volver.

Para entender

veo tus ojos y los de tu niña.

Nunca nos hablaron Malitzín,

de lo que significaba el regreso

Quizá nunca salimos.

Quizá nunca llegamos.

Incluso ahora,

dentro de las paredes de la casa,

Malitzín, 
buscamos huir

-o al menos lo sentíamos-

fundirnos hacinadas,

vientre a vientre,

cuando el frío nos viste

con lo único que tiene

Adoramos al tiempo,

aunque a veces se muestre incomprensible

Malitzín,

aunque a veces navegue

sin ancla por nuestra memoria.

Aunque no conozcamos ya nuestros nombres,

pero sí el signo de la que mira desde nosotras.

Entre rezos,

cera aterida que abraza otra cera aterida

la última vela del sahumerio es mi estancia.

(Solórzano, 2015, p. 51)

En 2007, Alejandra emprende un nuevo camino hacia Costa Rica, en donde entre otros asuntos estudió filosofía, publicó los dos libros a los cuales hago referencia en el presente trabajo y ejerce su profesión. En Costa Rica, Alejandra sigue siendo "Fiesta de pájaros/ Árbol invertido/ Enigma que espera en el patio de una abuela" (Solórzano, 2015, p. 58).

Debajo del silencio

la ecuación de la historia que debe inventarse en cada viaje.

Un país a cada tanto. El alma sobre el

cuaderno

Puta guerra.

Cierro los ojos dos segundos

(Mi madre y yo cenamos en silencio. Lanzo

una piedra para medir el fondo de un plato

de porcelana. Tengo 12 años)

El corazón

Sabe

Flotar. (Solórzano, 2017, p. 42) 


\section{Dolor, cuerpo, mujer y reencuentro. Acercamientos sensibles al sujeto migrante}

Escuchar a la persona, lo que el sujeto tiene que decir sin interpretar sus enunciados desde nuestras propias percepciones es una tarea imposible, construimos realidades intersubjetivamente, somos el conocimiento creado de manera colectiva en el transcurso de los siglos. Lo que sí es posible es escuchar sin objetivizar desde las pretensiones científicas, lo que el otro o la otra comunican.

Dolor, cuerpo, mujer y reencuentro son las cuatro impresiones que se me presentaron en la lectura de los textos de Alejandra, su poesía fue mi llave para acercarme al conocimiento sensible de estas cuatro manifestaciones del ser una mujer migrante.

\section{Dolor}

"Un nombre turbulento, una cicatriz ..." (Solórzano, 2015, p. 45).

Es reiterada la mención explícita a la tristeza y la cicatriz en esta selección de poemas de Alejandra (hecha por Alejandra), pero aún más que la mención explícita, los textos de la poeta están impregnados de un duelo que avanza con ella, un duelo itinerante como su vida. Alejandra no nos dice qué le duele aunque sí lo insinúa.

Qué puedo decirte

sino que este arpón hace de mí:

una trapecista

una pequeña campana sin nombre ... (Solórzano, 2015, p. 29)

... Remojá tus labios heridos

que se abra camino por tu cuerpo

el agua

-perfecta homicida-

salta obstáculos

abre impostoras cicatrices

cínica, insolente ... (Solórzano, 2017, p. 30)

El dolor de Alejandra es arpón que se nos atraviesa en el costado. Al leerla no tiene nombre concreto. Es el abandono de la tierra de origen. ¿Cuál tierra? ¿Guatemala? ¿Costa Rica? ¿Nicaragua? Es la lejanía de su padre y hermanas. ¿El desarraigo?, ¿los desarraigos? Es el dolor y la valentía de su madre. Es el dolor de una Centroamérica sangrante y violenta. Es una niña pequeña, que no entiende y que crece en la itinerancia "de tanto en tanto". 


\section{Buscarle}

con insistencia de cicatriz que persiste en el

mapa de

piel condenada al cambio, al movimiento.

La promesa de mi padre y el último gesto

materno una heredad de madrugada.

...

(Solórzano, 2017, p. 69)

La "mordedura", al contrario de lo que dictan las determinaciones teóricas sobre los procesos migratorios, es más que lo relativo a las formas, medios o razones de la migración. La "mordedura” es la no razón, lo inexplicable del sentimiento mismo, en código zambraniano, el dolor está en la oscuridad y se comunica en sensaciones inteligibles, por inconcretas en el lenguaje, sensaciones reales y desgarradoras.

\section{Cuerpo}

Alejandra desobedeció en secreto a su madre. Una de las medidas de seguridad era no conservar cartas, fotografías o documentos relacionados con la correspondencia entre su padre (en la montaña) y familia. Alejandra no se deshizo de la última carta de su padre previo al retorno y durante el viaje en bus a Guatemala, guardó la carta entre el trozo de tela de su calzón y su vulva. Viajó aterrada y mucho tiempo después conservó la culpa de haber podido poner en riesgo la seguridad del viaje, si hubieran topado con militares que subieran al bus para interrogarlas o bajarlas. Alejandra me dice que también necesitaba sentir que regresaba en compañía de su padre.

Es el cuerpo el primer lugar de lucha, la primera trinchera desde donde se recibe la experiencia, no en vano son constantes las referencias a los pies y las piernas en los poemas de Alejandra, recordándonos ese tránsito constante.

$\cdots$

La distancia

una danza oscilante

es mi corazón de Pom,

humo en tormento y alivio.

El espejismo de mis piernas

que ese nombre quiebra e instaura al final de los pasillos.

...

(Solórzano, 2015, p. 45) 
Tus piernas -que descubrí con mi adolescencia

y el retorno-desobedientes.

Huiré con ellas sin poder controlarlas,

rígidas, torpes y colmadas de dolor hermosas piernas azules

correré como pueda, sin dirección

sin orden alguno

como suele ser la belleza del sueño.

(Solórzano, 2015, p. 18)

Se relaciona el cuerpo y sus órganos, “corazón”, "lengua”, vientre” con un constante vaivén, un baile doloroso. Alejandra se entiende como un cuerpo que se desplaza.

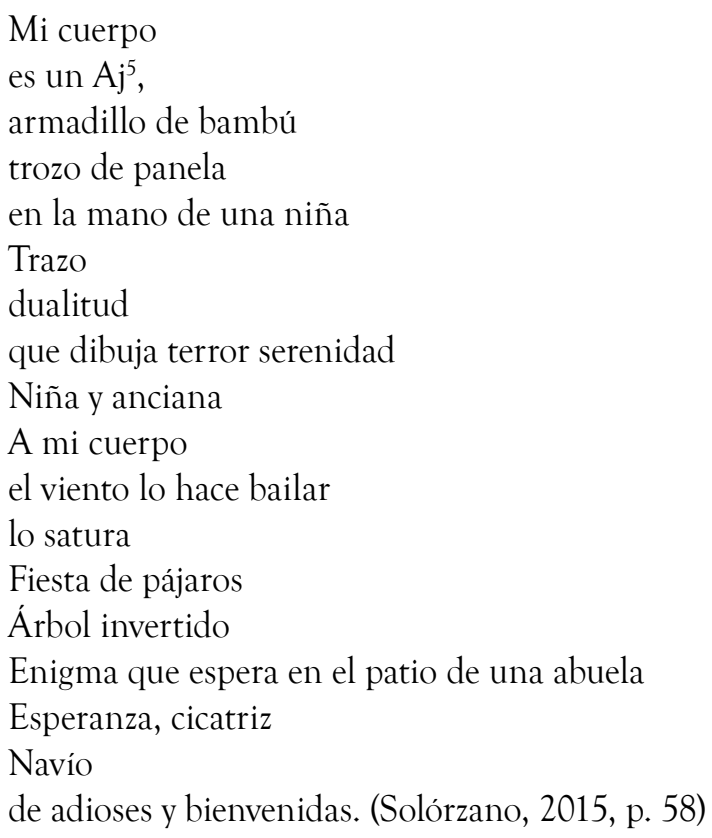

En el cuerpo se inscriben los enigmas y las cicatrices, en él se busca la propia historia, el transito transfronterizo, el paso de los días, las salidas y las llegadas.

5 Signo del calendario maya. 


\section{Mujer}

Alejandra le habla a Malitzín 6 , la entiende, le pregunta, la justifica y la abraza. Malitzín esa mujer que ha contado mil historias, ahora pareciera tener un rostro concreto en la poesía de Alejandra. ¿Malitzín es acaso Rosa María, Amanda, Ixmucané o Ivonne, es quizá la misma Alejandra?

En efecto una mujer que vive sola

sin un escudo historiado

sin una historia de niños

no es madre ni mujer

sino un nombre híbrido que viene

impreso al pie de tu página.

Alda Merini

Palmearán la espalda de Rivera

por girar tu rostro en el lienzo.

Tu rostro

moneda

limpia, pequeña

de una sola cara.

Sin secreto,

deseada y antigua.

Tu rostro, capullo de doble valor.

Alguien ató a tus pies una sombra que no te pertenece

una falsa hermana

igual que el color azul

que sale a gritos por los ojos de tu niño.

Presiento que la noche va a lapidarte Malitzín

Alguien lanzará tu rostro

a una fuente que arde

para pedir la conquista

No era por los dioses ni los indios

Querían tus mil lenguas y no conformes el corazón de chirimía.

A vos y tu sombra cetrina. Y así como antes, abro en este tiempo las manos y pregunto: ¿Sabrá la noche lo que habrán de preguntarte? ¿Sabrá algo sobre ti esa pira de libros? Algo, sí. Algo...un primer recuerdo.

6 También conocida como la Malinche. 
La destrucción y la flor cempaxúchitl en una

misma mañana. (Solórzano, 2015, p. 61)

Malitzín es la mujer centroamericana, es la mujer migrante reducida al olvido. Son inagotables las violencias sufridas por las personas migrantes, fueron incontables las violaciones y agresiones sufridas por los pueblos centroamericanos durante las guerras de los años setenta y ochenta, más de todas estas violencias fueron las mujeres los principales objetos. La figura mítica de Malitzín, surge como consuelo y heroína, como refugio ancestralmente femenino.

Malitzín camino de fuego, mujer que duerme sobre preguntas: ¿Dónde dejaste la última palabra de este siglo que fue mi insignia?

¿Dónde deberé buscarla, eco del cabello de mis abuelas? (Solórzano, 2015, p. 62)

\section{Reencuentro}

Esta es quizá la impresión más importante de los textos de Alejandra, el reencuentro es la constante y el motivo del movimiento y la poesía. ¿Por qué se mueve una persona migrante? ¿Está siempre en su intención el volver? ¿Regresar sana la "mordedura" de la partida? Estas preguntas tienen tantas respuestas como migrantes en el mundo. Por lo que no es posible una respuesta generalizada a partir de la experiencia de Alejandra; sin embargo, ese sentir subjetivo de constante movimiento y de anhelo de regreso a un país de origen, que ya no se conoce, es fundamental para entender los procesos de interculturalidad y adaptación de las personas migrantes en los países de tránsito o recepción.

El retorno es un espejo borroso Malitzín, una siempreviva petrificada, un viento preso.

…

Para entender veo tus ojos y los de tu niña.

Nunca nos hablaron Malitzín, de lo que significaba el regreso

Quizá nunca salimos.

Quizá nunca llegamos.

...

(Solórzano, 2015, p. 51) 


\section{Dos encargos}

Poder dar una mirada a la historia de Centroamérica, desde la poesía de Alejandra, genera una empatía superior a la que se puede obtener de la lectura de los datos históricos, demográficos y estadísticos. En los textos de Alejandra podríamos encontrar las sensaciones de la historia de cualquier persona, sea migrante o no, y, por tanto, no es difícil identificarse con la autora.

Las historias personales, sean contadas desde la formalidad de un género literario, o como relatos testimoniales, son las narraciones sentidas de los contextos sociohistóricos.

La historia oficial perpetúa la violencia e invisibiliza los hechos concretos, las cicatrices que guardan memorias. El primer encargo es el imperativo ético que tenemos desde los círculos académicos en generar espacios fónicos para quienes nunca han tenido vos, por lo que, en la búsqueda de caminos metodológicos para la dignificación, igualdad y justicia entre las personas, debemos mirar de manera sensible, sensorial y creativa hacia la subjetividad de las historias. Esto se trata de ir más allá de la visibilización de la desigualdad, se trata de involucrar todos los sentidos en la escucha atenta de las historias.

Tal ruta de subjetividades nos hace mirar las diferencias y las particularidades, lo heterogéneo de la realidad social. Por lo tanto, no podemos pasar por alto que tal y como se observa en la historia y la poesía de Alejandra, son las mujeres las principales receptoras de la violencia en los procesos migratorios, las guerras y la pobreza sistemática. El segundo encargo exige mirar, de manera especial, la particularidad y heterogeneidad de las historias de las mujeres, lo cual, probablemente, ofrezca un universo de significaciones invaluables para la comprensión sensible de la realidad. Es largo el trayecto recorrido por los feminismos y las mujeres en este encargo, son importantes los logros, pero aún hay mucho camino por delante y, por suerte, muchas formas de transitarlo.

\section{Conclusión}

Ser amiga de Alejandra es una bendición ${ }^{7}$ ¿ ¿Cuántas bendiciones más podemos evidenciar?

7 A falta de palabras en español uso bendición como: Privilegio, dicha, ruta y hecho. 


\section{Referencias}

Gonzalez, H., \& Rocha, J. (2009).Migración internacional en Centroamerica. Servicio Jesuita para Migrantes de Centro América. Universidad de Managua. https://doi.org/10.2307/j. ctv3dnq5h.8

Guerra, J. A. (2008). La razón poética. Una aproximación a María Zambrano y Heidegger. Aurora. Papeles de María Zambrano, 9. https://doi.org/10.1344/aurora2016.17.1

Herrera, J. ( s. f.) La reinvención de los derechos humanos. Atrapa Sueños. https://doi.org/10.2307/j.ctvt6rm4w.12

León, E. (2009). El giro hermenéutico de la fenomenológica en Martín Heidegger. Polis, 22. https:// journals.openedition.org/polis/2690

Martín, F. J. (1998). El sueño creador de María Zambrano (Razón poética y hermenéutica literaria). En J. Romera Castillo (Ed.), Sogno escrittura nelle culture iberiche. Atti del XVII Convegno AISPI (231-242). Bulzoni.

Morin, E. (2002). Con la Cabeza Blen Puesta. Repensar la reforma. Reformar el pensamiento. Nueva Visión.

Proyecto Estado de la Región. (1999). Primer informe estado de la región en desarrollo humano sostenible. (Capitulo XIV). https://doi.org/10.32418/rfs.2009.254.2027

Solórzano, A. (2015). Detener la historia. Ediciones Espiral.

Solórzano, A. (2017). Todo esto sucederá siempre. Edicones Espiral.

Zambrano , M. (2003). Pensamiento y poesía en la vida española. http://www.biblioteca.org.ar/ libros/89756.pdf.

Zambrano, M. (2006). Filosofía y poesía. Fondo de Cultura Económica. https://felonita.files. wordpress.com/2009/05/filosofiaypoesia.pdf 
